\title{
Bank Failures Caused by Large Withdrawals: An Explanation Based Purely on Liquidity*
}

\author{
Guilherme Carmona \\ Faculdade de Economia, Universidade Nova de Lisboa \\ Campus de Campolide, 1099-032 Lisboa, Portugal \\ email: gcarmona@fe.unl.pt \\ telephone: (351) 213801671 \\ fax: (351) 213886073
}

February 6, 2007

\footnotetext{
*This paper has circulated under the title "Bank Runs without Consumers' Panic" and "On the Existence of Equilibrium Bank Runs in a Diamond-Dybvig Environment". Special thanks are due to Patrick Leoni, who co-authored an early paper on this topic. I would also like to thank Bernardino Adão, Pedro Amaral, Alexandre Baptista, Aleksander Berentsen, Saltuk Ozerturk, Erwan Quintin, João Rosal, Karl Shell, and seminar participants at Southern Methodist University and Universität Basel for their comments. I thank also John Huffstot for editorial assistance. Any errors are mine.
} 


\begin{abstract}
In a version of the Diamond and Dybvig [8] model with aggregate uncertainty, we show that there exists an equilibrium with the following properties: all consumers deposit at the bank, all patient consumers wait for the last period to withdraw, and the bank fails with strictly positive probability. Furthermore, we show that the probability of a bank failure remains bounded away from zero as the number of consumers increases.

This equilibrium explains bank failures driven by extreme withdrawals solely on liquidity since they happen because both banks and depositors are illiquid. Furthermore, it does not require many of the elements typically emphasized, including: consumers well informed about the true state of nature, a non-zero consumption after a crisis, consumers' panic and sunspots. We therefore think that aggregate risk in DiamondDybvig-like environments can be an important element to explain bank crises.
\end{abstract}

Journal of Economic Literature Classification Numbers: C72, G21.

Keywords: Bank crises, aggregate uncertainty, liquidity. 


\section{Introduction}

Throughout history, we observe many episodes of a large number of people withdrawing their deposits from a bank, forcing it to fail. Since the work of Diamond and Dybvig [8], these episodes are understood as runs to illiquid banks: in a bank run, many or all of the bank's depositors attempt to withdraw their funds simultaneously, even those who are not in need of funds; these depositors want to withdraw because banks are illiquid and expected to fail due to these large withdrawals.

An alternative explanation of bank failures caused by large withdrawals is simply that they are caused by too many people in need of liquidity. While this explanation also depends on the (lack of) liquidity of banks, it does not require those who do not need funds to withdraw. In fact, this explanation depends purely on liquidity: these bank failures occur because both banks and depositors are illiquid.

We show that bank failures caused by large withdrawals can occur as an equilibrium phenomenon even without the economic elements traditionally emphasized as leading to bank runs. This establishes the above pure liquidity view as a sound theoretical explanation of bank failures caused by large withdrawals.

More precisely, we consider an environment similar to the one in Diamond and Dybvig [8]. The economy lasts for three periods. Consumers, who are all alike ex-ante, can be of two distinct types: impatient consumers, who need to consume early, and patient consumers, willing to postpone consumption. ${ }^{1}$

In contrast to those authors, we assume that there is a finite number of consumers, although this number will increase to infinity. We assume that there is aggregate uncertainty, modeled in the following way: first, the probability of each consumer being impatient is chosen according to a continuous density function; then, the consumers' type is determined

\footnotetext{
${ }^{1}$ As in their paper, we assume that the relative risk aversion coefficient exceeds one, but only for consumption levels above one (which is all that is needed for their results). In contrast to them, we assume that the utility of zero consumption is finite - obviously, otherwise there could not be a bank failure in equilibrium.
} 
in an i.i.d. way. As in Diamond and Dybvig, the consumers' type is their own private information. Furthermore, we assume that although each consumer knows his type, he does not know the true value of the probability of each consumer being impatient.

There are two technologies: storage with a constant rate of return and a productive technology whose rate of return dominates that of storages in the long run and is dominated in the short run. This implies that, in the absence of some financial asset, impatient consumers will receive a lower rate of return than do patient consumers. Thus, the main economic problem is how to share the risk of being an impatient consumer.

Risk sharing can be provided by banks. Consumers can deposit their initial endowment in a bank, or invest it directly. The banking system is assumed to be competitive and banks therefore offer contracts to depositors in order to maximize their ex-ante welfare.

Regarding the contracts that banks offer to consumers, we assume that the bank can only make payments contingent on whether it has failed (in which case it pays nothing) or not. ${ }^{2}$ Although this assumption can be (partially) justified by assuming that the only verifiable information is whether or not the bank has failed, ${ }^{3}$ our motivation is to assume it is to study whether or not it is possible to have bank failures driven by illiquid depositors when banks offer contracts akin to those observed in practice.

Our first main result then shows that if the population size is sufficiently high and if a sufficiently high fraction of impatient consumers is possible to occur, ${ }^{4}$ then there exists an

\footnotetext{
${ }^{2}$ We assume that consumers are isolated from each other, which implies the same trading restrictions (i.e., that depositors cannot sell their position at the bank) as in Diamond and Dybvig [8]. The importance of these trading restrictions has been analyzed by Jacklin [17].

${ }^{3}$ One can consider the framework of Andolfatto and Nosal [4] and consider the case of partial costless verification, where the verifiable information is whether or not the bank has failed. Specifically, we could assume that a monetary authority provides such information and punishes the bank when it falsely claims to have failed. However, this story provides only partial justification for our assumption since, as an anonymous referee has pointed out, it raises the question of why does the monetary authority not use those punishments to design more sophisticated contracts, such as the one considered in Green and Lin [13, 14].

${ }^{4}$ i.e., if the support of density function determining the probability of each consumer being impatient contains values sufficiently close to one.
} 
equilibrium in which every consumer deposits, no patient consumer withdraws early and the bank fails with a strictly positive probability.

In the above equilibrium, the bank failure corresponds to a situation where the fraction of impatient consumers is high. In such a case, a large number of depositors wants to withdraw, forcing the bank to fail. Since no patient consumer withdraws, this failure is not due to a bank run, but rather is caused by an extreme need of liquidity. This extreme need of liquidity leading to bank failures can be the result, for example, of poor economic conditions, as suggested by the empirical work of Gorton [12], Kaminsky and Reinhart [15] and Calomiris and Mason [6].

The probability of having a large number of impatient consumers, and therefore that of a bank failure, depends on both the realized and the expected number of impatient consumers (the latter being equal to the probability of each consumer being impatient). By the law of large numbers, in large economies only the second effect is important. Therefore, we might have a positive, but vanishing probability of a bank failure, in which case the importance of the purely liquidity-driven bank failures we are emphasizing would be negligible. Our second main result shows that this is not the case: it establishes, under the same assumptions, that the probability of a bank run is bounded away from zero as the population size increases to infinity.

A positive and non-vanishing probability of a bank failure in equilibrium is due to conjunction of illiquid banks and illiquid consumers. Furthermore, it depends on how illiquid the bank is, since the higher is the interest offered, the higher is the probability of a bank failure. In fact, we can rephrase our second main result as showing that the interest offered by the bank is higher and bounded away from 1 .

More precisely, consider a large economy, in which we are mainly interested. By the law of large numbers, as the number of consumers increases, the fraction of impatient depositors in the population converges to the probability of each one being impatient. In the limit, if the support of the density function determining the probability of each consumer being 
impatient is $[\underline{t}, \bar{t}]$, the bank will fail with a positive probability whenever it offers an interest rate higher than $\bar{r}=1 / \bar{t}$. Our second main result shows that the interest offered by the bank is higher and bounded away from $\bar{r}$. This implies that the desire to offer liquidity and a better risk sharing to impatient consumers is sufficiently high to compensate for the risk of a bank failure, in which some consumers will not be able to consume.

As we have illustrated above, most of our analysis is done by studying what happens in the limit as the number of consumers increases to infinity. In this way, our paper provides an example of Hildenbrand's [16] approach to study large economies with independent individual risks and in which per capita uncertainty vanishes. As is normally the case, there is no limit economy to which the sequence of finite economies we consider converges. Nevertheless, we show that all the functions used to define both the consumer's and bank's problem in the finite economies converge uniformly and that the law of large numbers holds. From the analysis of the limit problem, we are able to deduce the conclusions of our main results.

Of course, we could have simply analyzed the limit problem, which is rather similar to the problem considered by Diamond and Dybvig. The advantage of explicitly having a sequence of finite economies is that, although inferred from the study of a limit economy, our results cannot be regarded as an artifact of an infinite population. ${ }^{5}$

\section{Related Literature}

Regarding the main results of the paper, our inspiration comes from Wallace [23, p. 12], where he writes: "In my model, the cause of a bank run and a partial suspension is exogenous - an aggregate shock to tastes that makes the number of people wanting to withdraw unusually large." As in Wallace's work, a bank failure, although not a bank run, will occur here when the number of impatient consumers is high. Our results have two other important differences compared with Wallace's: first, bank crises have more severe consequences in our

\footnotetext{
${ }^{5}$ See Barlo and Carmona [5], Carmona [7] and Meirowitz [18] for more on this issue.
} 
results, since they cause banks to fail, whereas in Wallace's they lead to a partial suspension; second, Wallace's result relies on the assumption that there is a small amount of aggregate risk limited to a small group of individuals, which is not needed in ours — we believe that our result allows us to think of bank failures as a large-scale, society-wide phenomenon. ${ }^{6}$

A similar conclusion was established in Williamson [25]. In his model, banks are large coalitions of agents that write optimal contracts contingent on the state of nature. The allocation obtained in an equilibrium with banking coalition improves, in some states of nature, upon the allocation that would arise through decentralized capital markets, thus providing a rationale for banks. In some other states, associated with low output, both equilibria yield the same allocation, in which case one can expect that the bank will dissolve (say, to save on organizational costs). The main difference between these and our results is that, while bank failures in our framework have the same features as in [25] (namely, both output and utility are lower than average and deposit withdrawals are greater than average), they are also characterized by promises to pay that are not kept.

Our results are also related to those in Goldstein and Pauzner [11]. Here, as there, the probability of a bank failure increases in the degree of risk sharing offered by the bank but, despite such probability, the allocation so obtained improves consumers' welfare relative to autarky. Unlike their model, bank failures in our framework are not panic-based.

An important aspect of our results is that they allow bank failures driven by large withdrawals to occur in the presence of many elements that make them costly to consumers and/or difficult to occur in equilibrium. These include: (1) absence of consumers' panic, (2) absence of sunspots, (3) absence of mixed strategies, (4) sequential service constraint (and so zero consumption to late withdrawers), (5) an infinite marginal utility of consumption at zero, (6) absence of information about the true state of the economy, (7) recoverability of investment in the productive technology, and (8) a non-negligible probability of a high fraction of impatient consumers occurring.

\footnotetext{
${ }^{6}$ See Peck and Shell [20] and Ennis and Keister [9] for a similar point.
} 
We will comment briefly on each of these elements: Since all patient consumers prefer to wait, there is no consumers' panic in the bank failures of our model, an element that was important in Diamond and Dybvig [8], Peck and Shell [19] and Goldstein and Pauzner [11], among others. Typically, consumers' panic in equilibrium is obtained using sunspots, which are a crucial element for consumers to prefer to deposit despite the fact that the bank may fail (again, see Diamond and Dybvig [8] and Peck and Shell [19]). We emphasize that our results do not require sunspots.

We restrict consumer choice to pure strategies. Without this assumption one can show the existence of a positive probability of a bank run, and of a bank failure, as in Adão and Temzelides [1].

We also require banks to satisfy a sequential service constraint (which implies that, after it fails, late withdrawers will receive zero consumption). At the same time, we allow for an infinite marginal utility of consumption at zero, which together with the previous assumptions makes bank failures costly to those consumers who are unable to withdraw.

We assume that consumers have no information about the true state of the economy, and that the investment in the productive technology can be recovered - without these two assumptions, one can generate bank failures (and even bank runs) in which consumers run when the state of the economy is bad, as in Allen and Gale [2]. Furthermore, as has been shown by Goldstein and Pauzner [11], the same conclusion holds even if investment can be recovered and consumers have imperfect information about the true state of the economy.

Finally, we allow for a non-negligible probability of a high fraction of impatient consumers occurring. This increases the risk of a bank failure, which is costly. All of this suggests that the type of bank crises we address are quite robust in our Diamond and Dybvig framework. 


\section{The model}

In this section we formally describe the model. The model presented here is similar to that in Diamond and Dybvig [8].

There are three periods $T=0,1,2$. There is a single consumption good. There are two technologies: first, the consumer good can be stored from one period to the other; the gross rate of return equals 1 . Second, there is a productive technology, by which one unit invested in period 0 yields $R$, with $R>1$, units of consumption in period 2; furthermore, if the investment is interrupted in period 1, it will yield one unit (i.e., the investment can be recovered).

There is a finite number of consumers denoted by $n \in \mathbb{N}$. All consumers are identical in period 0. Consumers receive an endowment of one unit of consumption good in period 0 and zero in the remaining periods.

Each consumer can be of two distinct types, denoted by type 1 and type 2. A type 1 consumer values consumption in period 1 only (impatient consumer), whereas a type 2 consumer values consumption only in period 2 (patient consumer): Let $c_{1}$ denote the individual consumption received by a consumer in period 1 , let $c_{2}$ denote individual consumption received in period 2, and let $\Theta$ be the type of the agent. The utility derived by every agent from the consumption of the bundle $\left(c_{1}, c_{2}\right)$ is

$$
U\left(c_{1}, c_{2}, \Theta\right)= \begin{cases}u\left(c_{1}\right) & \text { if } \Theta=1 \\ u\left(c_{1}+c_{2}\right) & \text { otherwise }\end{cases}
$$

where $u: \mathbb{R}_{+} \rightarrow \mathbb{R}$ is twice continuously differentiable, strictly increasing and strictly concave. Furthermore, we assume:

Assumption $1 \quad$ 1. $-c u^{\prime \prime}(c) / u^{\prime}(c)>1$ for $c \geq 1$;

2. $u(0)=0$.

Every agent is assumed to maximize the ex-ante (relative to period 0) expected utility 
$E\left[U\left(c_{1}, c_{2}, \Theta\right)\right]^{7}$

The type of each consumer is determined by nature in 1 as follows: first, a number in $t \in[0,1]$ is drawn according to a probability measure $\mu$; then each consumer's type is drawn in an i.i.d. way with a probability $t$ of being of type 1 . It follows that the fraction of impatient consumers equals $i \in S_{n}=\{k / n: k=0, \ldots, n\}$ with probability $p_{n, t}(i)=t^{n i}(1-t)^{n-n i}$ when there are $n$ consumers and the probability of each being a type 1 is $t$.

Each consumer knows his own type, but not the type of the others; i.e., consumers' type is their own private information. Furthermore, no consumer knows the realized value of $t$.

We make the following assumptions on the uncertainty:

Assumption $2 \quad$ 1. The support of $\mu$ is an interval contained in $\left[0, t^{\prime}\right]$, where $0<t^{\prime} \leq 1$;

2. there exists a continuous function $f: \operatorname{supp}(\mu) \rightarrow \mathbb{R}_{+}$such that $\mu(B)=\int_{B} f$ for every Borel measurable set $B \subseteq[0,1]$.

The continuity of the density $f$ implies that if $\bar{t}=\max \{t: t \in \operatorname{supp}(\mu)\}$, then $F(t)=$ $\int_{0}^{t} f<1$ for all $t<\bar{t}$. Given any $\bar{t} \in[0,1]$, we write $f \in \mathcal{F}_{\bar{t}, B, x}$ when $f$ is continuous, bounded by $B>0, \bar{t}=\max \{t: t \in \operatorname{supp}(\mu)\}$ and $x=\int_{0}^{1} t f(t) \mathrm{d} t$.

We next describe the banking industry. There is a representative bank behaving competitively. The bank offers to the depositors a contract specifying a fixed claim of $r$ per ${ }^{7}$ An example of a function satisfying all of the above assumptions is $u(c)=-\exp ^{-2 c}+1$. In this case,
we would have $\lim _{c \rightarrow 0} u^{\prime}(c)=2$. An example of a function satisfying $\lim _{c \rightarrow 0} u^{\prime}(c)=\infty$ can be obtained as
follows: let
\[ v(c)= \begin{cases}\sqrt{c} & \text { if } c \leq 1 / 2, \\ -\frac{\sqrt{2} c^{-1}}{4}+\sqrt{2} & \text { otherwise. }\end{cases} \] The function $v$ is continuous in $[1 / 4, \infty)$ and smooth in $B=[1 / 4,1 / 2-\varepsilon] \cup[1 / 2+\varepsilon, \infty)$, where $0<\varepsilon<1 / 4$. Then, there exists a smooth function $\tilde{v}:[1 / 4, \infty) \rightarrow \mathbb{R}$ such that $\tilde{v}_{/ B}=v_{/ B}$. Then, let

$$
u(c)= \begin{cases}\sqrt{c} & \text { if } 0 \leq c \leq 1 / 2-\varepsilon, \\ \tilde{v}(c) & \text { if } 1 / 2-\varepsilon<c<1 / 2+\varepsilon, \\ -\frac{\sqrt{2} c^{-1}}{4}+\sqrt{2} & \text { if } c>1 / 2+\varepsilon .\end{cases}
$$


unit deposited to agents withdrawing in period 1 . The bank is mutually owned, and it is liquidated in period 2. This implies that period 2 withdrawers will share the remainder of the bank's assets equally among themselves.

If and when the bank fails, consumers will be informed (say, by a regulatory entity). The bank is closed and the remaining assets are distributed in period 2 to those claiming them. We assume that no impatient consumer will claim anything in period 2; in any case, an impatient consumer is indifferent between claiming or not, and so we can justify this assumption by postulating a positive cost of exercising it.

Thus, the amounts received by depositors are as follows: Let $A$ denote the total amount deposited in period 0 and $r$ be the interest rate offered by the bank. Consider a depositor $j$ willing to withdraw in period 1 and let $l_{j}$ denote the number of withdrawers arriving at the bank before consumer $j$. Then $j$ 's period 1 payoff is equal to $r$ if $r\left(l_{j}+1\right) \leq A$ and 0 otherwise. That is, if the bank can fully pay him, he will receive $r$. In contrast, if $r\left(l_{j}+1\right)>A$, then the bank is closed and the remaining assets (equal to $A-r l_{j}$ ) are kept for period $2 .^{8}$

If a depositor waits for period 2 to withdraw, then he receives

$$
\max \left\{\frac{R(A-r l)}{h}, 0\right\}
$$

where $l$ denotes the number of depositors who have withdrawn in period 1 and $h$ denotes the number of those that have not withdrawn in period 1 and want to withdraw in period 2. Since we assume that no impatient consumer wants to withdraw in period $2, h$ equals the number of patient consumers who have not withdrawn in period 1 .

We assume that consumers are isolated from each other during period 1, although each one contacts the bank at some point in that period. As Wallace [22] has shown, this implies that the bank has to satisfy a sequential service constraint; that is, the bank must serve the depositors withdrawing in period 1 in the (random) order that they arrive at the bank until

\footnotetext{
${ }^{8}$ Alternatively, we could assume that depositor $j$ receives $A-r l_{j}$, but this would only complicate our analysis.
} 
it runs out of assets. We assume that all orderings are equally likely, and so each occurs with a probability of $1 / m$ ! when there are $0 \leq m \leq n$ withdrawers. In order to evaluate different strategies, each consumer needs to know the probability of arriving at the bank before it fails. If the bank can fully pay $k$ depositors, then the probability that a given consumer is fully paid equals

$$
\alpha\left(\frac{m}{n}, \frac{k}{n}\right)=\min \left\{1, \frac{k(m-1) !}{m !}\right\}=\min \left\{1, \frac{k}{m}\right\}=\min \left\{1, \frac{k / n}{m / n}\right\} .
$$

In the above definition of $\alpha$ we use as arguments the fraction of depositors trying to withdraw and the fraction of depositors that can be fully paid in period 1. In general, this probability depends on the interest rate $r$ offered by the bank and on the strategies chosen by the other consumers. We let $k_{n}(r)$ satisfy $r k_{n}(r) \leq 1$ and $r\left(k_{n}(r)+1 / n\right)>1$; thus, $n k_{n}(r)$ is the number of depositors that can be fully paid in period 1 when the bank offers $r$ and all consumers deposit.

A strategy of the bank is the choice of $r \in[1, R]$; the bank chooses $r$ in order to maximize ex-ante utility of the consumers (recall that they are equal ex-ante). This behavior is motivated by the competitive nature of the banking industry. ${ }^{9}$ More generally, we could assume that the bank offers $r \in \mathbb{R}$, but risk-aversion will ensure that $r \in[1, R]$. The bank chooses the interest rate in period 0 and then announces it to the consumers.

In period 0, each consumer will choose whether to deposit given the interest rate offered by the bank; those who do not deposit will invest in the productive technology. For simplicity, we assume that consumers have to deposit all of the endowment. ${ }^{10}$ In period 1 each consumer learns her type and then chooses either to withdraw from the bank or to wait, depending on her type, on the interest rate and on her deposit choice. Consumers who withdraw their deposit in period 1 can either consume the goods received or store

\footnotetext{
${ }^{9}$ Adão and Temzelides [1] have shown in a similar framework that another type of bank's behavior is possible in equilibrium; however, bank's maximization of the ex-ante utility of the consumers is the only behavior plausible in more refined notions of equilibrium.

${ }^{10}$ In fact, our results extend to the case where consumers are allowed to deposit just a fraction of their endowment.
} 
them. ${ }^{11}$ Hence, a strategy for a consumer is $(d, w)$, where $d$ is a function from $[1, R]$ into $\{0,1\}$, and $w$ is a function from $[1, R] \times\{0,1\} \times \Theta$ into $\{0,1\}$. We make the convention that $d(r)=1$ stands for the choice of depositing, and similarly $w(r, d, \Theta)=1$ means that she will withdraw in period $1 .^{12}$

A symmetric equilibrium is then $r^{*}, d^{*}$, and $w^{*}$ such that $w^{*}(r, d, \Theta)$ is optimal for all $(r, d, \Theta), d^{*}(r)$ is optimal for all $r$, and $r^{*}$ is optimal taking as given agents' strategies.

The bank fails in the first period if $d^{*}\left(r^{*}\right)=1$ and $1<r^{*} m / n$ where $r^{*}$ is an equilibrium interest rate and $m$ is the number of depositors that choose to withdraw (i.e., $w^{*}\left(r^{*}, 1, \Theta\right)=$ 1). That is, the total value of assets that depositors plan to withdraw in period 1 strictly exceeds the total value of assets owned by the bank, also in period 1.

We say that an equilibrium bank failure occurs asymptotically with positive probability if there exists $N \in \mathbb{N}$ such that $n \geq N$ implies the existence of a symmetric equilibrium with the following properties: (1)every consumer deposits (i.e., $\left.d^{*}\left(r^{*}\right)=1\right),(2)$ all patient consumers wait (i.e., $\left.w^{*}\left(r^{*}, d^{*}\left(r^{*}\right), 2\right)=0\right)$ and (3) the bank fails in the first period with strictly positive probability.

In the above equilibrium there is a bank failure caused by large withdrawals in the sense that a large number of depositors go to the bank, causing it to fail. However, since all patient consumers prefer to wait, it follows that there is no consumers' panic.

\section{Equilibrium Bank Failures}

In this section, we consider whether equilibrium bank failures caused by large withdrawals exist and how robust they are. In Section 4.1, we give a sufficient condition that guarantees the existence of equilibrium bank failures with positive probability in large economies. Then, in Section 4.2, we show that under the same assumption, the probability of a bank failure is bounded away from zero as the number of consumers increases.

\footnotetext{
${ }^{11}$ This implies that storage will only be helpful for patient consumers who withdraw early.

${ }^{12}$ Note that we allow only pure strategies. It is important to note that it is possible to have bank runs in a mixed strategy equilibrium, as shown by Adão and Temzelides [1].
} 


\subsection{Existence of Equilibrium Bank Failures}

In an equilibrium bank failure caused by large withdrawals, all consumers choose to deposit and patient consumers prefer to wait. So, it is necessary to guarantee the existence of equilibria with these properties, which one can do under the assumptions made in Section 2 .

We can construct such an equilibrium by studying a particular maximization problem. This problem consists of choosing an interest rate $r$ in order to maximize consumers' ex-ante utility among those that make consumers prefer to deposit and patient consumers to wait, given that everyone else is depositing and every other patient depositor is waiting.

We describe this problem below, which is indexed by the number of consumers. The ex-ante expected utility if all follow the above strategy can be obtained as follows: if a consumer is of type 1 , he receives $r$ with probability $\alpha\left(i, k_{n}(r)\right)$ when the fraction of impatient consumers is $i$ and the bank can fully pay $n k_{n}(r)$ consumers. If the consumer is of type

2 , he receives $\max \left\{0, \frac{R(n-r i)}{n-i}\right\}$. Thus, letting $U_{n}(r)$ denote the ex-ante expected utility, we have

$$
\begin{aligned}
U_{n}(r)= & \int_{0}^{1} f(t)\left[t \sum_{i \in S_{n-1}} p_{n-1, t}(i) \alpha\left(\frac{(n-1) i+1}{n}, k_{n}(r)\right) u(r)\right. \\
& \left.+(1-t) \sum_{i \in S_{n-1}} p_{n-1, t}(i) u\left(\max \left\{0, \frac{R(n-r(n-1) i)}{n-(n-1) i}\right\}\right)\right] \mathrm{d} t .
\end{aligned}
$$

In particular, suppose that the bank offers an interest rate equal to $r$, all consumers deposit, and all patient consumers wait for the second period to withdraw. Then, the expected utility for a patient consumer equals

$$
\int_{0}^{1} f(t) \sum_{i \in S_{n-1}} p_{n-1, t}(i) u\left(\max \left\{0, \frac{R(n-r(n-1) i)}{n-(n-1) i}\right\}\right) \mathrm{d} t .
$$

If one patient consumer decides to withdraw in period 1 , then his expected utility, when all the other patient consumers withdraw in period 2, is given by

$$
\int_{0}^{1} f(t) \sum_{i \in S_{n-1}} p_{n-1, t}(i) \alpha\left(\frac{(n-1) i+1}{n}, k_{n}(r)\right) u(r) \mathrm{d} t .
$$


Thus, letting $W_{n}=\left\{r \in[1, R]: W L_{n}(r) \geq W R_{n}(r)\right\}$, where $W L_{n}(r)$ is defined by equation (7) and $W R_{n}(r)$ by equation (8), we have that any patient consumer will choose to withdraw in period 2 provided that $r$ belongs to $W_{n}$, all consumers deposit, and all other patient consumers wait for period 2 to withdraw.

If a consumer decides not to deposit, his ex-ante expected utility is simply

$$
\int_{0}^{1} f(t)[t u(1)+(1-t) u(R)] \mathrm{d} t
$$

which is equal to $U_{n}(1)$. Hence, letting $D_{n}=\left\{r \in[1, R]: U_{n}(r) \geq U_{n}(1)\right\}$, we see that any consumer will choose to deposit provided that $r$ belongs to $D_{n}$, all other consumers deposit, and all patient consumers wait for period 2 to withdraw.

Thus, consider the following problem:

$$
\begin{aligned}
& \max _{r \in[1, R]} U_{n}(r) \\
& \text { subject to } r \in W_{n} \cap D_{n},
\end{aligned}
$$

One can show that this problem has a solution (see Lemma 11 in the Appendix). Using the solution to this problem, it is then easy to construct an equilibrium in which all consumers deposit and all patient consumers wait (see Lemma 12).

Although the existence of such equilibria is necessary for our purposes, it is not enough. Without further assumptions, the interest rate offered by the bank in such equilibria may be equal to one, in which case there will be no bank failure. Thus, we need additional assumptions in order to guarantee that bank failures occur with positive probability. Essentially, we need the support of $f$ to have values sufficiently close to one, and a large population.

Proposition 1 For every $B>0$ and $x \in(0,1)$ there exists $\tau \in[0,1]$ such that if $\tau<$ $\bar{t}<1$ and $f \in \mathcal{F}_{\bar{t}, B, x}$ then an equilibrium bank failure occurs asymptotically with positive probability.

One important element needed for Proposition 1 is a large population. By the law of large numbers, the fraction of impatient consumers in the population converges to the 
probability of each consumer being impatient as the size of the population increases. This implies that in the limit there is only one source of aggregate uncertainty, which is the one represented by $f$. This is in contrast to what happens in any finite economy, where some aggregate uncertainty stems from the consumers' idiosyncratic preference shocks. This is important partly because the probability that the fraction of impatient consumers is between $\bar{t}$ and 1 is always positive in any finite economy, but is zero in the limit.

The above comment suggests that the analysis of a limit problem where the law of large numbers holds might be useful and easier. In the Appendix A.1 we show that problem (9) converges, in the sense that all the functions involved converge uniformly, to the following problem:

$$
\begin{aligned}
& \max _{r \in[1, R]} U(r)=\int_{0}^{1} f(t)\left[t \alpha(t, k(r)) u(r)+(1-t) u\left(\max \left\{0, \frac{R(1-r t)}{1-t}\right\}\right)\right] \mathrm{d} t \\
& \text { subject to } r \in W \cap D,
\end{aligned}
$$

where $k(r)=1 / r, D=\{r \in[1, R]: U(r) \geq U(1)\}$ and

$$
W=\left\{r \in[1, R]: \int_{0}^{1} f(t) u\left(\max \left\{0, \frac{R(1-r t)}{1-t}\right\}\right) \mathrm{d} t \geq \int_{0}^{1} f(t) \alpha(t, k(r)) u(r) \mathrm{d} t\right\} .
$$

In this problem, the variable $t$ can be thought of as the fraction of impatient consumers in the population. In this way, it is very similar to the one considered initially by Diamond and Dybvig [8].

The analysis of the limit problem above reveals that its solution exceeds $\bar{r}=1 / \bar{t}$, provided that $\bar{t}$ is sufficiently close to 1 . This allows us to conclude that the solution to problem (9) is also greater than $\bar{r}$ if the population is large enough, leading directly to a positive probability of a bank failure. This reasoning illustrates why we need the support of $f$ to have values sufficiently close to one.

\subsection{A Limit Result on the Probability of Bank Failures}

As we have pointed out above, there are two sources of aggregate uncertainty in a finite economy. This implies that there are two reasons for having a positive probability of bank 
failure due to large withdrawals whenever the bank offers an interest rate greater than 1. First, for any possible $t>0$, the probability that the fraction of impatient consumers is greater than $1 / r$ is always positive - this probability has to do with the distribution associated with $p_{n, t}$. A second reason has to do with the distribution associated with $f$, which can also (by the law of large numbers) be thought of as representing the probability that the fraction of impatient consumers is greater than $1 / r$.

Clearly, the second effect alluded to above can only take place if $r$ exceeds $\bar{r}$. If this is not the case, then the probability of a bank failure is essentially due only to the consumers' idiosyncratic shocks, and would vanish as the population size increases. What Proposition 2 below shows is that this is not the case: in fact, it shows that part of a positive probability of bank failures comes from the desire to provide a better risk sharing, which is expressed in an interest rate greater and bounded away from $\bar{r}$.

We now turn to Proposition 2. Under the conditions of Proposition 1, we know that asymptotically there exist equilibrium bank failures. For every $n$ sufficiently large, let $\left(r_{n}^{*}, d_{n}^{*}, w_{n}^{*}\right)$ be an equilibrium in which there is a bank run without consumers' panic. Let $\gamma_{n}$ be the corresponding probability of a bank run.

Proposition 2 The sequence $\left\{\gamma_{n}\right\}_{n}$ is bounded away from 0.

Proposition 2 shows that no matter how large the economy is, and thus no matter how well the idiosyncratic risk is diversified, the probability of a bank failure never goes below a certain value. This implies that an economy can experience a bank failure due to large withdrawals even if no consumer panics, since these bank failures have a fundamental nature: they occur when the average fraction of impatient consumers is high, i.e., when there are many people who need liquidity.

At this point we can provide an easy illustration of why it is necessary that the support of $f$ has values sufficiently close to one: if $R=2$ and $\bar{t}=1 / 3$, then $\bar{r}=1 / \bar{t}=3>R$. Thus, an upper bound for $\gamma_{n}$ is obtained when the bank offers $r_{n}=R$ for all $n$, which we denote by $\tilde{\gamma}_{n}$. It follows easily from the law of large numbers that in this case $\tilde{\gamma}_{n}$ converges to zero. 
Thus, we need $\bar{r}$ to be large.

\section{Concluding Remarks}

We used the standard Diamond and Dybvig [8] framework to show that bank failures caused by large withdrawals can be explained as the result of illiquid banks and illiquid consumers, without many of the elements emphasized in the literature. In our version of the Diamond and Dybvig framework, such bank failures will occur whenever there is a large number of depositors in need of short-term funds. These are possible because banks will choose to offer a high short-term interest rate in order to provide better risk sharing for their depositors; however, the interest rate offered is sufficiently high to lead to a positive probability that the bank will not have enough funds to pay all early withdrawers. Hence, bank failures of this type are a direct consequence of the degree of banks' liquidity. Furthermore, our construction is such that the probability of a bank run is sufficiently small to guarantee that those who do not need funds early will prefer not to withdraw early. We thus depart from the idea that in an extreme withdrawing episode some depositors withdraw when they do not need to - this is the sense in which such episodes should not be regarded as bank runs.

The type of equilibria on which we concentrate reflects some practical features of the banking system: banks offer liquidity and risk sharing to depositors, people deposit and withdraw only when they have to, and sometimes banks fail. Furthermore, an equilibrium of this type has the property of having no consumer panic, and still bank failures occur with a positive, bounded away from zero, probability.

Although not explicitly modeled, we may interpret the aggregate uncertainty over the number of early withdrawers as reflecting business cycle conditions. For instance, we expect the number of people who need short-term funding to be influenced by the unemployment rate - this will be the case as long as unemployed individuals try to compensate the loss of income by using their assets to smooth out consumption. We then expect that fundamental 
shocks that lead to a large number of early withdrawers can lead to extreme withdrawals. Therefore, our results are consistent with the business cycle view of bank crises, a view that has received some empirical support (see Gorton [12], Kaminsky and Reinhart [15] and Calomiris and Mason [6]). This suggests that explicitly introducing the type of fundamental shocks studied in the business cycle literature might be promising, a challenge we take up in Amaral and Carmona [3]. 


\section{A Appendix}

In this appendix, we will prove our results. In Section A.1, and following Hildenbrand [16], we will start by showing that problem (9) converges to the type of problem studied by Diamond and Dybvig [8] as the number of consumers goes to infinity. Although we are interested in finite economies, the latter problem is interesting because it is easy to study. This is essentially because the law of large numbers holds.

In Section A.2, we study the limit problem. The main result there (Lemma 7) shows that the interest rate offered by the bank is sufficiently high to allow for bank failures due to large withdrawals in the limit problem under certain conditions. ${ }^{13}$ The main difficulty regarding this result has to do with the possibility that the marginal utility of consumption is not bounded from above; therefore, much of the effort is devoted to showing that standard limit results, such as the Lebesgue's dominated convergence theorem is still applicable.

In Section A.3, we show that for every $n \in \mathbb{N}$ ( $n$ denotes the population size) there exists an equilibrium in which all consumers deposit at the bank and all patient consumers wait to withdraw. Finally, and under the same conditions as in Lemma 7, we show in Section A.4 that there is a positive probability of an equilibrium bank run in large finite economies; furthermore, we show in Section A.5 that such a probability of an equilibrium bank run remains bounded away from zero as the number of consumers increases.

\section{A.1 The Limit Problem}

Lemma 1 For any continuous function $h:[\alpha, \beta] \times[a, b] \rightarrow \mathbb{R}$, with $[a, b] \subseteq[0,1]$,

$$
\int_{[a, b]} h(r, i) \mathrm{d} p_{n, t}(i) \rightarrow h(r, t)
$$

uniformly in $r$ and $t$.

Proof. Let $\varepsilon>0$. Since $h$ is continuous in $[\alpha, \beta] \times[a, b]$, a compact set, $h$ is bounded. Let $B>\varepsilon$ be such that $|h| \leq B$ and let $\delta>0$ be such that $|x-y|<\delta$ implies that

\footnotetext{
${ }^{13}$ For an illustration of the idea of Lemma 7, see the last column of Table 1 in Ennis and Keister [9].
} 
$|h(x)-h(y)|<\varepsilon / 2$. Then, since $p_{n, t}\left(B_{\delta}(t)\right) \geq 1-\frac{t(1-t)}{n \delta^{2}}$ (see Freund [10, p. 190]), it follows that

$$
\begin{aligned}
& \left|\int_{[a, b]} h(r, i) \mathrm{d} p_{n, t}(i)-h(r, t)\right| \leq\left|\int_{B_{\delta}(t)} h \mathrm{~d} p_{n, t}-p_{n, t}\left(B_{\delta}(t)\right) h(r, t)\right|+ \\
& \left|\int_{B_{\delta}^{c}(t)} h \mathrm{~d} p_{n, t}-p_{n, t}\left(B_{\delta}^{c}(t)\right) h(r, t)\right| \leq p_{n, t}\left(B_{\delta}(t)\right) \frac{\varepsilon}{2}+p_{n, t}\left(B_{\delta}^{c}(t)\right) 2 B \leq \\
& \left(1-\frac{t(1-t)}{n \delta^{2}}\right) \frac{\varepsilon}{2}+\frac{t(1-t)}{n \delta^{2}} 2 B \leq\left(1-\frac{1}{4 n \delta^{2}}\right) \frac{\varepsilon}{2}+\frac{1}{4 n \delta^{2}} 2 B<\varepsilon,
\end{aligned}
$$

if $n$ is sufficiently large. Thus, $\sup _{(r, t)}\left|\int_{[a, b]} h(r, i) \mathrm{d} p_{n, t}(i)-h(r, t)\right| \leq \varepsilon$ for $n$ sufficiently large, which completes the proof.

Lemma 2 Let $h$ and $h_{n}$, for all $n \in \mathbb{N}$, be real-valued functions on $[\alpha, \beta] \times[a, b]$, with $[a, b] \subseteq[0,1]$, satisfying $h$ is continuous and $h_{n}$ converges uniformly to $h$. Then,

$$
\int_{[a, b]} h_{n}(r, i) \mathrm{d} p_{n, t}(i) \rightarrow h(r, t)
$$

uniformly on $r$ and $t$.

Proof. Let $\varepsilon>0$. Then, since

$$
\left|\int_{[a, b]} h_{n}(r, i) \mathrm{d} p_{n, t}(i)-\int_{[a, b]} h(r, i) \mathrm{d} p_{n, t}(i)\right| \leq \int_{[a, b]}\left|h_{n}-h\right| \mathrm{d} p_{n, t} \leq \sup _{(r, i)}\left|h_{n}(r, i)-h(r, i)\right|,
$$

it follows by lemma 1 that

$$
\begin{aligned}
\left|\int_{[a, b]} h_{n}(r, i) \mathrm{d} p_{n, t}(i)-h(r, t)\right| \leq & \left|\int_{[a, b]} h_{n}(r, i) \mathrm{d} p_{n, t}(i)-\int_{[a, b]} h(r, i) \mathrm{d} p_{n, t}(i)\right|+ \\
& \left|\int_{[a, b]} h(r, i) \mathrm{d} p_{n, t}(i)-h(r, t)\right|<\varepsilon,
\end{aligned}
$$

if $n$ is sufficiently large. Thus, $\sup _{(r, t)}\left|\int_{[a, b]} h_{n}(r, i) \mathrm{d} p_{n, t}(i)-h(r, t)\right| \leq \varepsilon$ for $n$ sufficiently large, which completes the proof.

Define, for $t, i \in[0,1]$,

$$
V_{n}^{1}(r, i)=\alpha\left(i+\frac{1-i}{n}, k_{n}(r)\right) u(r),
$$


and

$$
V_{n}^{2}(r, i)=u\left(\max \left\{0, \frac{R\left(1-r\left(1-\frac{1}{n}\right) i\right)}{1-\left(1-\frac{1}{n}\right) i}\right\}\right) .
$$

Also let $k(r)=1 / r$,

$$
V^{1}(r, i)=\alpha(i, k(r)) u(r)
$$

and

$$
V^{2}(r, i)=u\left(\max \left\{0, \frac{R(1-r i)}{1-i}\right\}\right)
$$

Lemma 3 1. $k_{n}(r)$ converges to $k(r)$ uniformly;

2. $\alpha\left(i+(1-i) / n, k_{n}(r)\right)$ converges to $\alpha(i, k(r))$ uniformly in $i \in[0,1]$ and $r \in[1, R]$;

3. $t V_{n}^{1}(r, i)+(1-t) V_{n}^{2}(r, i)$ converges uniformly to $t V^{1}(r, i)+(1-t) V^{2}(r, i)$ in $i \in[0,1-\varepsilon]$ and $r \in[1, R]$ for all $\varepsilon>0$;

4. $(r, i) \mapsto t V^{1}(r, i)+(1-t) V^{2}(r, i)$ is continuous when $i \in[0,1-\varepsilon]$ and $r \in[1, R]$ for all $\varepsilon>0$ and $t \in[0,1]$.

Proof. 1. We have that

$$
k(r)-\frac{1}{n} \leq k_{n}(r) \leq k(r)
$$

which implies that $\left|k_{n}(r)-k(r)\right| \leq 1 / n$ and so, $k_{n}(r)$ converges uniformly to $k(r)$. Equation (19) can be established as follows: if $k_{n}(r)>k(r)$, then $r k_{n}(r)>r k(r)=1$, a contradiction; if $k_{n}(r)<k(r)-1 / n$, then $r\left(k_{n}(r)+1 / n\right)<r k(r)=1$, a contradiction.

2. We start by noting the following fact: if $(r, i) \mapsto a_{n}(r, i)$ converges uniformly (in $i$ and $r)$ to $(r, i) \mapsto a(r, i),(r, i) \mapsto b_{n}(r, i)$ converges uniformly to $(r, i) \mapsto b(r, i)$, both $a$ and $b$ are bounded and $b$ and $b_{n}$ are bounded away from zero (i.e., there is $\eta>0$ such that $b(r, i) \geq \eta$ and $b_{n}(r, i) \geq \eta$ for all $(r, i)$ and all $\left.n\right)$, then $a_{n} / b_{n}$ converges uniformly to $a / b$.

Suppose that $i \geq 1 / r$. Then $\alpha(i, k(r))=1 /(r i)$ and since $k(r) \geq k_{n}(r)$ and $i+(1-i) / n \geq$ $i$, it follows that $\alpha\left(i+(1-i) / n, k_{n}(r)\right)=k_{n}(r) /(i+(1-i) / n)$. In order to apply the above fact, let $a_{n}(r, i)=k_{n}(r), a(r, i)=1 / r, b_{n}(r, i)=i+(1-i) / n$ and $b(r, i)=i$. Since 
all the conditions are satisfied (in particular, $b_{n}(r, i) \geq b(r, i) \geq 1 / R$ ), it follows that $\sup _{(r, i): r i \geq 1}\left|\alpha\left(i+(1-i) / n, k_{n}(r)\right)-\alpha(i, k(r))\right|$ converges to zero.

Finally, suppose that $i<1 / r$. Then, $k(r)=1$ and so

$$
\begin{aligned}
& \left|\alpha\left(i+(1-i) / n, k_{n}(r)\right)-\alpha(i, k(r))\right|=1-\min \left\{1, \frac{k_{n}(r)}{i+\frac{1-i}{n}}\right\} \leq \\
& 1-\frac{k_{n}(r)}{1+1 / n} \leq 1-\frac{k(r)-1 / n}{1+1 / n}=1-\frac{1-1 / n}{1+1 / n} .
\end{aligned}
$$

Thus, $\sup _{(r, i): r i<1}\left|\alpha\left(i+(1-i) / n, k_{n}(r)\right)-\alpha(i, k(r))\right| \rightarrow 0$. Hence, $\alpha\left(i+(1-i) / n, k_{n}(r)\right)$ converges to $\alpha(i, k(r))$ uniformly in $r$ and $i$.

3. It follows from part 2 that $V_{n}^{1}(r, i)$ converges uniformly to $V^{1}(r, i)$.

It remains to show that $V_{n}^{2}(r, i)$ converges uniformly to $V^{2}(r, i)$ if $r \in[1, R]$ and $i \in$ $[0,1-\varepsilon]$, with $\varepsilon>0$. Define

$$
\beta_{n}(r, i)=\max \left\{0, \frac{R\left(1-r\left(1-\frac{1}{n}\right) i\right)}{1-\left(1-\frac{1}{n}\right) i}\right\}
$$

and

$$
\beta(r, i)=\max \left\{0, \frac{R(1-r i)}{1-i}\right\}
$$

Since $\beta_{n}(1, i)=\beta(1, i)=R$, we may assume that $r>1$. Note also that $\beta_{n}(r, i) \geq \beta(r, i)$.

If $i \leq 1 / r$ then $\beta(r, i) \geq 0$, and we readily see that all the conditions of the fact in part 2 of this proof are satisfied: let $a(r, i)=R(1-r i), a_{n}(r, i)=R(1-r i(1-1 / n)), b(r, i)=1-i$ and $b_{n}(r, i)=1-i(1-1 / n)$; in particular $b_{n} \geq b \geq \varepsilon$.

If $i>1 / r$, then $\beta(r, i)=0$ and since $\beta_{n}(r, i)$ is decreasing in $i$, we obtain

$$
\begin{aligned}
\left|\beta_{n}(r, i)-\beta(r, i)\right| & \leq \frac{R\left(1-r\left(1-\frac{1}{n}\right) \frac{1}{r}\right)}{1-\left(1-\frac{1}{n}\right) \frac{1}{r}}=R \frac{1 / n}{1-1 / r+1 /(r n)} \\
& \leq R \frac{1 / n}{1-1 / r} \leq \frac{R}{\varepsilon n} \rightarrow 0 .
\end{aligned}
$$

Thus, $\beta_{n}(r, i)$ converges uniformly to $\beta(r, i)$. Since $u$ is continuous, then $V_{n}^{2}(r, i)$ converges uniformly to $V^{2}(r, i)$.

\section{Obvious.}

Let

$$
U(r)=\int_{0}^{1} f(t)\left[t \alpha(t, k(r)) u(r)+(1-t) u\left(\max \left\{0, \frac{R(1-r t)}{1-t}\right\}\right)\right] \mathrm{d} t
$$


and $k(r)=1 / r$.

Lemma 4 Let $B>0, x \in(0,1), 0<\bar{t}<1$ and $f \in \mathcal{F}_{\bar{t}, B, x}$. Then, $U_{n}(r)$ converges uniformly to $U(r)$.

Proof. We may write $U_{n}(r)=\int_{0}^{\bar{t}} f(t) V_{n}(r, t) \mathrm{d} t$, where

$$
V_{n}(r, t)=\int_{0}^{1}\left(t V_{n}^{1}(r, i)+(1-t) V_{n}^{2}(r, i)\right) \mathrm{d} p_{n-1, t}(i) .
$$

We may also define

$$
V(r, t)=t \alpha(t, k(r)) u(r)+(1-t) u\left(\max \left\{0, \frac{R(1-r t)}{1-t}\right\}\right)
$$

and write $U(r)=\int_{0}^{\bar{t}} f(t) V(r, t) \mathrm{d} t$. Thus, in order to prove the lemma, it is enough to show that $V_{n}$ converges uniformly to $V$ for $r \in[1, R]$ and $t \in[0, \bar{t}]$.

Let $0<\eta<1-\bar{t}$. Then,

$$
\left|V_{n}(r, t)-V(r, t)\right| \leq\left|\int_{0}^{\bar{t}+\eta} V_{n}(r, i) \mathrm{d} p_{n-1, t}(i)-V(r, t)\right|+\left|\int_{\bar{t}+\eta}^{1} V_{n}(r, i) \mathrm{d} p_{n-1, t}(i)\right| .
$$

Since $p_{n-1, t}\left(B_{\eta}(t)\right) \geq 1-\frac{t(1-t)}{(n-1) \eta^{2}}$ (see Freund [10, p. 190]), we have that

$$
\begin{aligned}
& \left|\int_{\bar{t}+\eta}^{1} V_{n}(r, i) \mathrm{d} p_{n-1, t}(i)\right| \leq u(R) p_{n-1, t}([\bar{t}+\eta, 1]) \leq \\
& u(R) p_{n-1, t}([0, t-\eta] \cup[t+\eta, 1]) \leq u(R) \frac{t(1-t)}{(n-1) \eta^{2}} \leq u(R) \frac{1}{4(n-1) \eta^{2}} .
\end{aligned}
$$

Since $u(R) /\left[4(n-1) \eta^{2}\right]$ converges to zero and is independent of $r$ and $t$, it remains to show that

$$
\left|\int_{0}^{\bar{t}+\eta} V_{n}(r, i) \mathrm{d} p_{n-1, t}(i)-V(r, t)\right|
$$

converges to zero uniformly. This follows from Lemma 2 and 3.

Let

$$
W L(r)=\int_{0}^{1} f(t) u\left(\max \left\{0, \frac{R(1-r t)}{1-t}\right\}\right) \mathrm{d} t
$$

and

$$
W R(r)=\int_{0}^{1} f(t) \alpha(t, k(r)) u(r) \mathrm{d} t .
$$


Lemma 5 Let $B>0, x \in(0,1), 0<\bar{t}<1$ and $f \in \mathcal{F}_{\bar{t}, B, x}$. Then, $W L_{n}(r)$ converges uniformly to $W L(r)$ and $W R_{n}(r)$ converges uniformly to $W R(r)$.

Proof. Analogous to Lemma 4.

\section{A.2 Analysis of the Limit Problem}

Let $D=\{r \in[1, R]: U(r) \geq U(1)\}$ and $W=\{r \in[1, R]: W L(r) \geq W R(r)\}$.

Lemma 6 The function $U$ has a maximizer in $D \cap W$.

Proof. Note that the set $D \cap W$ is compact, and non-empty, since $r=1$ belongs to $D \cap W$. The function $U$ is a continuous function of $r$. Hence, there exists $r^{*}$ that maximizes $U$ in $D \cap W$.

Lemma 7 For all $B>0$ and $x \in(0,1)$, there is $\tau \in[0,1]$ such that if $\tau<\bar{t}<1$ and $f \in \mathcal{F}_{\bar{t}, B, x}$ the following holds:

There exists $\tilde{r}>1 / \bar{t}$ such that $U(\tilde{r})>U(r)$ for all $1 \leq r \leq 1 / \bar{t}$ and $W L(\tilde{r})>W R(\tilde{r})$.

Lemma 7 is the key lemma to establish Proposition 1. Before presenting its proof, we need some technical lemmas.

Lemma $8 \int_{0}^{r} u^{\prime}=u(r)$ for any $r>0$.

Proof. Let $0<\delta<r$. Then $\int_{\delta}^{r} u^{\prime}=u(r)-u(\delta)$ (see Wheeden and Zygmund [24, Theorem 5.52, p.83] and Rudin [21, Theorem 6.21, p. 134]).

Define $h_{k}=\chi_{[1 / k, r]} u^{\prime}$ for all $k \in \mathbb{N}$. Since $h_{k} \geq 0$ for all $k$, and $h_{k} \nearrow u^{\prime}$, it follows that $\int_{0}^{r} h_{k} \rightarrow \int_{0}^{r} u^{\prime}$. Hence, $\int_{0}^{r} u^{\prime}=\lim _{k}[u(r)-u(1 / k)]=u(r)-u(0)=u(r)$.

Let $g(r, t)=\frac{R(1-r t)}{1-t}$.

Lemma 9 If $r>1$ and $0 \leq c<d \leq 1 / r$, then

$$
\int_{c}^{d} u^{\prime}(g(r, t)) \mathrm{d} t=\int_{\frac{R(1-r d)}{1-d}}^{\frac{R(1-r c)}{1-c}} u^{\prime}(y) \frac{R(r-1)}{(R r-y)^{2}} \mathrm{~d} y .
$$


Proof. We start by noting the following extension of the change of variable theorem (Rudin $[21$, Theorem 6.19 , p. 132]): if $\gamma:[A, B] \rightarrow[b, a]$ is a strictly decreasing continuous function with $\gamma^{\prime}$ Riemann integrable and $h$ is Riemann integrable on $[b, a]$, then $\int_{b}^{a} h(x) \mathrm{d} x=$ $\int_{A}^{B}\left(-\gamma^{\prime}(y)\right) h(\gamma(y)) \mathrm{d} y$

Assume first that $d<1 / r$. Let $\gamma(y)=\frac{R-y}{R r-y}$. Letting $g_{r}(t)=g(r, t)$, we see that $\gamma=g_{r}^{-1}$. Then $-\gamma^{\prime}(y)=\frac{R(r-1)}{(R r-y)^{2}}$, and is therefore bounded by $1 / R(r-1)($ since $y \leq R)$. Thus, by the above,

$$
\int_{c}^{d} u^{\prime} \circ g(r, t) \mathrm{d} t=\int_{c}^{d} u^{\prime} \circ g_{r}(t) \mathrm{d} t=\int_{\frac{R(1-r d)}{1-d}}^{\frac{R(1-r c)}{1-c}} u^{\prime}(y) \frac{R(r-1)}{(R r-y)^{2}} \mathrm{~d} y .
$$

If $d=1 / r$, using a limit argument similar to that in Lemma 8 , we can show that

$$
\int_{c}^{1 / r} u^{\prime}(g(r, t)) \mathrm{d} t=\lim _{\delta \rightarrow 1 / r} \int_{c}^{\delta} u^{\prime}(g(r, t)) \mathrm{d} t=\int_{0}^{\frac{R(1-r c)}{1-c}} u^{\prime}(y) \frac{R(r-1)}{(R r-y)^{2}} \mathrm{~d} y .
$$

We can now proceed with the proof of Lemma 7.

Proof of Lemma 7. Since $W L(1)=u(R)>u(1)=W R(1)$, then there exists $\zeta>0$ such that $1<r<1+\zeta$ implies $W L(r)>W R(r)$.

Let $f$ be such that $\int_{0}^{1} t f(t) \mathrm{d} t=x$. Consider the following function $M:[0,1] \rightarrow \mathbb{R}$ defined by

$$
M(r)=\int_{0}^{1}\left[u^{\prime}(r)-R u^{\prime}(g(r, t))\right] t f(t) \mathrm{d} t .
$$

Clearly, $M(1)=\left[u^{\prime}(1)-R u^{\prime}(R)\right] \int_{0}^{1} t f(t) \mathrm{d} t=\left[u^{\prime}(1)-R u^{\prime}(R)\right] x$ and so $M(1)>0$ (see Diamond and Dybvig [8, footnote 2]).

Let $\left\{\bar{t}_{k}\right\}_{k} \subset(0,1)$ be such that $\lim _{k} \bar{t}_{k}=1$ and $\left\{f_{k}\right\}$ be a sequence of densities belonging to $\mathcal{F}_{\bar{t}_{k}, B, x}$, but otherwise arbitrary. Let $\bar{r}_{k}=1 / \bar{t}_{k}$, for all $k$.

Claim $1 \lim _{k} \int_{0}^{1}\left[u^{\prime}\left(\bar{r}_{k}\right)-R u^{\prime}\left(g\left(\bar{r}_{k}, t\right)\right)\right] t f_{k}(t) \mathrm{d} t=\left[u^{\prime}(1)-R u^{\prime}(R)\right] x$.

Proof. Since $u^{\prime}$ is continuous, we have that $u^{\prime}\left(\bar{r}_{k}\right) x \rightarrow u^{\prime}(1) x$; thus, it remains to show that $\int_{0}^{1} u^{\prime} \circ g\left(\bar{r}_{k}, t\right) t f_{k}(t) \mathrm{d} t \rightarrow x u^{\prime}(R)$. Note that $x u^{\prime}(R)=\int_{0}^{1} u^{\prime} \circ g(1, t) t f_{k}(t) \mathrm{d} t$ for all $k$. 
We have that $u^{\prime} \circ g\left(\bar{r}_{k}, t\right) \geq u^{\prime}(R)=u^{\prime} \circ g(1, t)$ for all $t \in\left[0, \bar{t}_{k}\right]$ and so

$$
\begin{aligned}
& \left|\int_{0}^{1} u^{\prime} \circ g\left(\bar{r}_{k}, t\right) t f_{k}(t) \mathrm{d} t-\int_{0}^{1} u^{\prime} \circ g(1, t) t f_{k}(t) \mathrm{d} t\right| \\
& \leq B \int_{0}^{1}\left(u^{\prime} \circ g\left(\bar{r}_{k}, t\right)-u^{\prime} \circ g(1, t)\right) \chi_{\left[0, \bar{t}_{k}\right]} \mathrm{d} t .
\end{aligned}
$$

Since $\int_{0}^{1} u^{\prime} \circ g(1, t) \chi_{\left[0, \bar{t}_{k}\right]} \mathrm{d} t \rightarrow u^{\prime}(R)=\int_{0}^{1} u^{\prime} \circ g(1, t) \mathrm{d} t$, it is enough to show that

$$
\int_{0}^{\bar{t}_{k}} u^{\prime} \circ g\left(\bar{r}_{k}, t\right) \mathrm{d} t=\int_{0}^{1} u^{\prime} \circ g\left(\bar{r}_{k}, t\right) \chi_{\left[0, \bar{t}_{k}\right]} \mathrm{d} t \rightarrow \int_{0}^{1} u^{\prime} \circ g(1, t) \mathrm{d} t .
$$

Clearly, $0 \leq u^{\prime} \circ g\left(\bar{r}_{k}, t\right) \chi_{\left[0, \bar{t}_{k}\right]} \rightarrow u^{\prime} \circ g(1, t)$ for all $t<1$. Let $\varepsilon>0$. Then, by the bounded convergence theorem (Wheeden and Zygmund [24, Corollary 5.37, p. 76]) $\int_{0}^{1-\varepsilon} u^{\prime} \circ g\left(\bar{r}_{k}, t\right) \mathrm{d} t \rightarrow u^{\prime}(R)(1-\varepsilon)$. By Lemma 9 , for $k$ large,

$$
\int_{1-\varepsilon}^{1} u^{\prime} \circ g\left(\bar{r}_{k}, t\right) \chi_{\left[0, \bar{t}_{k}\right]} \mathrm{d} t=\int_{1-\varepsilon}^{\bar{t}_{k}} u^{\prime} \circ g\left(\bar{r}_{k}, t\right) \mathrm{d} t=\int_{0}^{R\left(1-\bar{r}_{k}+\varepsilon \bar{r}_{k}\right) / \varepsilon} u^{\prime}(y) \frac{R\left(\bar{r}_{k}-1\right)}{\left(R \bar{r}_{k}-y\right)^{2}} \mathrm{~d} y .
$$

We have that

$$
\begin{aligned}
& \int_{0}^{R\left(1-\bar{r}_{k}+\varepsilon \bar{r}_{k}\right) / \varepsilon} u^{\prime}(y) \frac{R\left(\bar{r}_{k}-1\right)}{\left(R \bar{r}_{k}-y\right)^{2}} \mathrm{~d} y= \\
& \int_{0}^{1} u^{\prime}(y) \frac{R\left(\bar{r}_{k}-1\right)}{\left(R \bar{r}_{k}-y\right)^{2}} \mathrm{~d} y+\int_{1}^{R\left(1-\bar{r}_{k}+\varepsilon \bar{r}_{k}\right) / \varepsilon} u^{\prime}(y) \frac{R\left(\bar{r}_{k}-1\right)}{\left(R \bar{r}_{k}-y\right)^{2}} \mathrm{~d} y \leq \\
& \frac{R\left(\bar{r}_{k}-1\right)}{\left(R \bar{r}_{k}-1\right)^{2}} \int_{0}^{1} u^{\prime}(y) \mathrm{d} y+u^{\prime}(1) R\left(\bar{r}_{k}-1\right) \int_{1}^{R\left(1-\bar{r}_{k}+\varepsilon \bar{r}_{k}\right) / \varepsilon} \frac{1}{\left(R \bar{r}_{k}-y\right)^{2}} \mathrm{~d} y= \\
& u(1) \frac{R\left(\bar{r}_{k}-1\right)}{\left(R \bar{r}_{k}-1\right)^{2}}+u^{\prime}(1)\left[\frac{R\left(\bar{r}_{k}-1\right)}{R \bar{r}_{k}-R\left(1-\bar{r}_{k}+1\right)}=\right. \\
& u(1) \frac{R\left(\bar{r}_{k}-1\right)}{\left(R \bar{r}_{k}-1\right)^{2}}+u^{\prime}(1)\left[\varepsilon-\frac{R\left(\bar{r}_{k}-1\right)}{R \bar{r}_{k}-1}\right] \rightarrow \varepsilon u^{\prime}(1) .
\end{aligned}
$$

Thus,

$$
u^{\prime}(R) \leq \liminf _{k} \int_{0}^{\bar{t}_{k}} u^{\prime} \circ g\left(\bar{r}_{k}, t\right) \mathrm{d} t \leq \limsup _{k} \int_{0}^{\bar{t}_{k}} u^{\prime} \circ g\left(\bar{r}_{k}, t\right) \mathrm{d} t \leq \varepsilon u^{\prime}(1)+(1-\varepsilon) u^{\prime}(R),
$$

and so $\lim _{k} \int_{0}^{\bar{t}_{k}} u^{\prime} \circ g\left(\bar{r}_{k}, t\right) \mathrm{d} t=u^{\prime}(R)$.

Hence, it follows from claim 1 that there exists $0<\tau<1$ such that if $\tau<\bar{t}<1$ and $f \in \mathcal{F}_{\bar{t}, B, x}$, then $M_{f}(\bar{r})>0$. Furthermore, we can choose $\tau$ so that and $W L(r)>W R(r)$ for all $r \in[1, \bar{r}]$.

Let $\bar{t} \in(\tau, 1)$ and $f \in \mathcal{F}_{\bar{t}, B, x}$. Note that $U$ is concave in $[0, \bar{r}]$, where $\bar{r}=1 / \bar{t}$ as before. This follows from the fact that both $u$ and $r \mapsto u \circ g(r, t)$ are concave for all $t \in[0, \bar{r}]$ : we have that $\partial^{2} u \circ g(r, t) / \partial r^{2}=u^{\prime \prime} \circ g(r, t)(t R)^{2} /(1-t)^{2}<0$. 
Claim $2 U^{\prime}(r)=M(r)$ for all $r \in[1, \bar{r}]$.

Proof. Let $r \in(1, \bar{r}]$ and let $r_{k} \nearrow r$. Then

$$
\frac{U\left(r_{k}\right)-U(r)}{r_{k}-r}=\int_{0}^{\bar{t}} f(t)\left[t \frac{u\left(r_{k}\right)-u(r)}{r_{k}-r}+(1-t) \frac{u \circ g\left(r_{k}, t\right)-u \circ g(r, t)}{r_{k}-r}\right] \mathrm{d} t .
$$

So, it is enough to show that

$$
\int_{0}^{\bar{t}} f(t) t \frac{u\left(r_{k}\right)-u(r)}{r_{k}-r} \mathrm{~d} t \rightarrow \int_{0}^{\bar{t}} f(t) u^{\prime}(r) \mathrm{d} t
$$

and

$$
\int_{0}^{\bar{t}} f(t)(1-t) \frac{u \circ g\left(r_{k}, t\right)-u \circ g(r, t)}{r_{k}-r} \mathrm{~d} t \rightarrow \int_{0}^{\bar{t}} f(t) t R u^{\prime} \circ g(r, t) \mathrm{d} t .
$$

We have that $\left\{\left(u\left(r_{k}\right)-u(r)\right) /\left(r_{k}-r\right)\right\}_{k}$ is non-negative and decreasing and that $t \mapsto$ $t f(t)\left(u\left(r_{1}\right)-u(r)\right) /\left(r_{1}-r\right)$ is integrable. The desired convergence then follows from the monotone convergence theorem (see Wheeden and Zygmung [24, Theorem 5.32, p. 75]). Similarly, $\left\{\left(u \circ g\left(r_{k}, t\right)-u \circ g(r, t)\right) /\left(r_{k}-r\right)\right\}_{k}$ is non-positive and decreasing, and so $\{-(u \circ$ $\left.\left.g\left(r_{k}, t\right)-u \circ g(r, t)\right) /\left(r_{k}-r\right)\right\}_{k}$ is non-negative and increasing, and so the desired convergence follows also from the monotone convergence theorem.

The case $r \in[1, \bar{r})$ and $r_{k} \searrow r$ is analogous.

Since $U$ is concave, then $M$ is decreasing. This implies that $U(\bar{r}) \geq U(r)$ for all $1 \leq r \leq \bar{r}$ as follows: A necessary condition for a solution to the problem

$$
\begin{aligned}
& \max _{r \in[1, R]} U(r) \\
& \text { subject to } r \in W \cap D \text { and } r \leq \bar{r} .
\end{aligned}
$$

is that $[r-\bar{r}] M(r)=0$. Since $M(r) \geq M(\bar{r})>0$ for all $r \in[1, \bar{r}]$, it follows that $\bar{r}$ maximizes $U(r)$ in $[1, \bar{r}]$.

Finally, we claim that there exists $\tilde{r}>\bar{r}$ such that $U(\tilde{r})>U(\bar{r})$ and $W L(\tilde{r})>W R(\tilde{r})$.

Since $W L(\bar{r})>W R(\bar{r})$, we conclude that there exists a ball $B(\bar{r})$ around $\bar{r}$ such that $r \in B(\bar{r})$ implies $W L(r)>W R(r)$. Therefore, to prove the existence of $\tilde{r}$ with the above properties, it is enough to show that

$$
\liminf _{r \searrow \bar{r}} \frac{U(r)-U(\bar{r})}{r-\bar{r}}>0 .
$$


This is so, because if equation (36) holds, then it cannot be the case that $\frac{U(r)-U(\bar{r})}{r-\bar{r}} \leq 0$ for all $r>\bar{r}$ in the ball $B(\bar{r})$ around $\bar{r}$. This implies the existence of $\tilde{r}>\bar{r}$ in $B(\bar{r})$ such that $\frac{U(\tilde{r})-U(\bar{r})}{\tilde{r}-\bar{r}}>0 ;$ this, of course, implies that $U(\tilde{r})>U(\bar{r})$.

We have that

$$
\begin{aligned}
\frac{U(r)-U(\bar{r})}{r-\bar{r}}= & \int_{0}^{1 / r}\left[t \frac{u(r)-u(\bar{r})}{r-\bar{r}}+(1-t) \frac{u \circ g(r, t)-u \circ g(\bar{r}, t)}{r-\bar{r}}\right] f(t) \mathrm{d} t+ \\
& \frac{1}{r-\bar{r}} \int_{1 / r}^{\bar{t}}\{t[\alpha(t, k(r)) u(r)-u(\bar{r})]-(1-t) u \circ g(\bar{r}, t)\} f(t) \mathrm{d} t .
\end{aligned}
$$

Let $\left\{r_{k}\right\}_{k}$ be such that $r_{k} \searrow \bar{r}$. Note that $\alpha\left(t, k\left(r_{k}\right)\right)=1 /\left(t r_{k}\right)=t_{k} / t$, with $t_{k}=1 / r_{k}$. Let $\varepsilon>0$; if $k \in \mathbb{N}$ is sufficiently large, then we have that $\left|t_{k} u\left(r_{k}\right)-t u(\bar{r})\right|<\varepsilon$ for all $t \in\left[t_{k}, \bar{t}\right]$ since

$$
\begin{aligned}
\left|t_{k} u\left(r_{k}\right)-t u(\bar{r})\right| & \leq t_{k}\left|u\left(r_{k}\right)-u(\bar{r})\right|+u(\bar{r})\left|t_{k}-t\right| \\
& \leq \bar{t}\left|u\left(r_{k}\right)-u(\bar{r})\right|+u(\bar{r})\left|t_{k}-\bar{t}\right|
\end{aligned}
$$

and $t_{k} \rightarrow \bar{t}$. Therefore,

$$
\begin{aligned}
& \left|\frac{1}{r_{k}-\bar{r}} \int_{1 / r_{k}}^{\bar{t}} t\left[\alpha\left(t, k\left(r_{k}\right)\right) u\left(r_{k}\right)-u(\bar{r})\right] f(t) \mathrm{d} t\right|=\left|\frac{1}{r_{k}-\bar{r}} \int_{1 / r_{k}}^{1 / \bar{r}}\left[t_{k} u\left(r_{k}\right)-t u(\bar{r})\right] f(t) \mathrm{d} t\right| \leq \\
& \frac{\varepsilon}{r_{k}-\bar{r}} \int_{1 / r_{k}}^{1 / \bar{r}} f(t) \mathrm{d} t=\varepsilon \frac{F(1 / \bar{r})-F\left(1 / r_{k}\right)}{r_{k}-\bar{r}} \rightarrow \varepsilon \frac{f(1 / \bar{r})}{\bar{r}^{2}} .
\end{aligned}
$$

Since $\varepsilon$ is arbitrary, then

$$
\lim _{k} \frac{1}{r_{k}-\bar{r}} \int_{1 / r_{k}}^{\bar{t}}\{t[\alpha(t, k(r)) u(r)-u(\bar{r})] f(t) \mathrm{d} t=0 .
$$

Also, by the fundamental theorem of calculus,

$$
\frac{1}{r_{k}-\bar{r}} \int_{1 / r_{k}}^{1 / \bar{r}}(1-t) f(t) u \circ g(\bar{r}, t) \mathrm{d} t \rightarrow(1-\bar{t}) f(\bar{t}) u \circ g(\bar{r}, \bar{t}) / \bar{r}^{2}=0,
$$

since $g(\bar{r}, \bar{t})=0$ and $u(0)=0$. Hence, it remains to show that

$$
\liminf _{r \searrow \bar{r}} \int_{0}^{1 / r}\left[t \frac{u(r)-u(\bar{r})}{r-\bar{r}}+(1-t) \frac{u \circ g(r, t)-u \circ g(\bar{r}, t)}{r-\bar{r}}\right] f(t) \mathrm{d} t>0 .
$$

Define

$$
h_{k}(t)= \begin{cases}{\left[t \frac{u\left(r_{k}\right)-u(\bar{r})}{r_{k}-\bar{r}}+(1-t) \frac{u \circ g\left(r_{k}, t\right)-u \circ g(\bar{r}, t)}{r_{k}-\bar{r}}\right] f(t)} & \text { if } t \in\left[0,1 / r_{k}\right] \\ 0 & \text { otherwise }\end{cases}
$$


Also, let

$$
h_{k}^{1}=t \frac{u\left(r_{k}\right)-u(\bar{r})}{r_{k}-\bar{r}} \chi_{\left[0,1 / r_{k}\right]}
$$

and

$$
h_{k}^{2}=(1-t) \frac{u \circ g\left(r_{k}, t\right)-u \circ g(\bar{r}, t)}{r_{k}-\bar{r}} \chi_{\left[0,1 / r_{k}\right]} .
$$

Clearly, $\int_{0}^{1 / \bar{r}} h_{k}^{1} \rightarrow \int_{0}^{1 / \bar{r}} u^{\prime}(\bar{r}) t f(t) \mathrm{d} t$. Also, for all $\varepsilon>0, \int_{0}^{1 / \bar{r}} h_{k}^{2}=\int_{0}^{1 / \bar{r}-\varepsilon} h_{k}^{2}+\int_{1 / \bar{r}-\varepsilon}^{1 / \bar{r}} h_{k}^{2}$ and $\int_{0}^{1 / \bar{r}-\varepsilon} h_{k}^{2} \rightarrow-\int_{0}^{1 / \bar{r}-\varepsilon} R u^{\prime} \circ g(\bar{r}, t) t f(t) \mathrm{d} t$, since $h_{k}^{2}$ is bounded in $[0, \bar{t}-\varepsilon]$.

Consider $\int_{1 / \bar{r}-\varepsilon}^{1 / \bar{r}} h_{k}^{2}$. We have that

$$
\begin{aligned}
& \int_{1 / \bar{r}-\varepsilon}^{1 / \bar{r}} h_{k}^{2}=\int_{1 / \bar{r}-\varepsilon}^{1 / r_{k}}(1-t) \frac{u \circ g\left(r_{k}, t\right)-u \circ g(\bar{r}, t)}{r_{k}-\bar{r}} f(t) \mathrm{d} t \geq \\
& B \int_{1 / \bar{r}-\varepsilon}^{1 / r_{k}}(1-t) \frac{u \circ g\left(r_{k}, t\right)-u \circ g(\bar{r}, t)}{r_{k}-\bar{r}} \mathrm{~d} t=-B \int_{1 / \bar{r}-\varepsilon}^{1 / r_{k}}(1-t) u^{\prime} \circ g\left(c_{k, t}, t\right) \frac{t R}{1-t} \mathrm{~d} t \geq \\
& -B R \int_{1 / \bar{r}-\varepsilon}^{1 / r_{k}} u^{\prime} \circ g\left(c_{k, t}, t\right) \mathrm{d} t \geq-B R \int_{1 / \bar{r}-\varepsilon}^{1 / r_{k}} u^{\prime} \circ g\left(r_{k}, t\right) \mathrm{d} t,
\end{aligned}
$$

where the first inequality uses the fact that $0 \leq f \leq B$ and $u \circ g\left(r_{k}, t\right)<u \circ g(\bar{r}, t)$, the second uses the mean value theorem, and so $\bar{r} \leq c_{k, t} \leq r_{k}$, and the last uses the fact that $u^{\prime} \circ g$ is increasing and $c_{k, t} \leq r_{k}$.

Choose $\varepsilon>0$ such that $(R \bar{r} \varepsilon) /(1-\bar{t}+\varepsilon)<1$. Then, for $k$ large, $\left(R\left(1-r_{k}(\bar{t}-\varepsilon)\right)\right) /(1-$ $\bar{t}+\varepsilon)<1$, since $\bar{r} \bar{t}=1$. By Lemma 9 , it follows that

$$
\begin{aligned}
& \int_{1 / \bar{r}-\varepsilon}^{1 / \bar{r}} h_{k}^{2} \geq-B R \int_{0}^{\frac{R\left(1-r_{k}(\bar{t}-\varepsilon)\right)}{1-t+\varepsilon}} u^{\prime}(y) \frac{R\left(r_{k}-1\right)}{\left(R r_{k}-y\right)^{2}} \mathrm{~d} y \\
& \geq-B R \frac{R\left(r_{k}-1\right)}{\left(R r_{k}-1\right)^{2}} \int_{0}^{\frac{R\left(1-r_{k}(\bar{t}-\varepsilon)\right)}{1-t+\varepsilon}} u^{\prime}(y) \mathrm{d} y=-K_{k} u\left(\frac{R\left(1-r_{k}(\bar{t}-\varepsilon)\right)}{1-\bar{t}+\varepsilon}\right),
\end{aligned}
$$

where $K_{k}=B R \frac{R\left(r_{k}-1\right)}{\left(R r_{k}-1\right)^{2}}$. Note that the last equality follows from Lemma 8 .

Hence, it follows that

$$
\begin{aligned}
\liminf _{k} \int_{0}^{1 / r_{k}} h_{k} & \geq \int_{0}^{1 / \bar{r}-\varepsilon}\left[u^{\prime}(\bar{r})-R u^{\prime} \circ g(\bar{r}, t)\right] t f(t) \mathrm{d} t \\
& +\int_{1 / \bar{r}-\varepsilon}^{1 / \bar{r}} u^{\prime}(\bar{r}) t f(t) \mathrm{d} t-B R \frac{R(\bar{r}-1)}{(R \bar{r}-1)^{2}} u\left(\frac{R \bar{r} \varepsilon}{1-\bar{t}+\varepsilon}\right) .
\end{aligned}
$$

Since $\int_{0}^{1 / \bar{r}}\left[u^{\prime}(\bar{r})-R u^{\prime} \circ g(\bar{r}, t)\right] t f(t) \mathrm{d} t=M(\bar{r})>0$, it follows in particular that $0 \leq \int_{0}^{1 / \bar{r}} R u^{\prime} \circ$ $g(\bar{r}, t) t f(t) \mathrm{d} t<\infty$. Thus,

$$
\lim _{\varepsilon \rightarrow 0} \int_{0}^{1 / \bar{r}-\varepsilon} R u^{\prime} \circ g(\bar{r}, t) t f(t) \mathrm{d} t=\int_{0}^{1 / \bar{r}} R u^{\prime} \circ g(\bar{r}, t) t f(t) \mathrm{d} t
$$


and so

$$
\lim _{\varepsilon \rightarrow 0} \int_{0}^{1 / \bar{r}-\varepsilon}\left[u^{\prime}(\bar{r})-R u^{\prime} \circ g(\bar{r}, t)\right] t f(t) \mathrm{d} t=\int_{0}^{1 / \bar{r}}\left[u^{\prime}(\bar{r})-R u^{\prime} \circ g(\bar{r}, t)\right] t f(t) \mathrm{d} t .
$$

Since $\lim _{\varepsilon \rightarrow 0} u((R \bar{r} \varepsilon) /(1-\bar{t}+\varepsilon))=0$, it follows that

$$
\liminf _{k} \int_{0}^{1 / r_{k}} h_{k} \geq \int_{0}^{1 / \bar{r}}\left[u^{\prime}(\bar{r})-R u^{\prime} \circ g(\bar{r}, t)\right] t f(t) \mathrm{d} t=M(\bar{r})>0 .
$$

This completes the proof.

\section{A.3 Existence of a Non-Autarkic Equilibrium with a Finite Number of Consumers}

In this section we establish the existence of an equilibrium in which all consumers deposit at the bank.

Lemma 10 For any $n, U_{n}$ is upper semi-continuous.

Proof. Note that

$$
k_{n}(r)= \begin{cases}1 & \text { if } r=1 \\ \frac{n-j}{n} & \text { if } \frac{n}{n-j+1}<r \leq \frac{n}{n-j}, \quad j=1, \ldots, n-1 \\ 0 & \text { if } r>n\end{cases}
$$

Thus, $k_{n}$ is upper semi-continuous, and so is $U_{n}$.

Lemma 11 The program

$$
\begin{aligned}
& \max _{r \in[1, R]} U_{n}(r) \\
& \text { subject to } r \in W_{n} \cap D_{n},
\end{aligned}
$$

has a solution.

Proof. Let $I_{j}=\left[\frac{n}{n-j+1}, \frac{n}{n-j}\right]$ for $j=1, \ldots, n-1, I_{n}=[n, \infty)$ and $I_{0}=\{1\}$. For each $j=0, \ldots, n$, consider the following problem, denoted $P^{j}$ :

$$
\max _{r} U_{n}^{j}(r)=E_{t} E_{i}\left[\frac{n-j}{(n-1) i+1} u(r)+(1-t) u\left(\max \left\{\frac{R(n-r(n-1) i}{n-(n-1) i}\right\}\right)\right]
$$

subject to $r \in I_{j} \cap W_{n}^{j} \cap D_{n}$, 
where

$$
W_{n}^{j}=\left\{r \in[1, R]: W L(r) \geq \frac{n-j}{(n-1) i+1} u(r)\right\}
$$

for $j=1, \ldots, n$ and $W_{n}^{0}=W_{n}$. Let $S$ be the set of those $j$ s for which the constraint set is non-empty; the set $S$ is non-empty since $1 \in I_{1} \cap W_{n}^{0} \cap D_{n}$ and so $0 \in S$. Then, if $j \in S$, the above problem has a solution, since the objective function is upper semi-continuous, and the constraint set is compact. Let $r_{j}^{*}$ be a solution to $P^{j}$.

Since $U_{n}$ jumps down if (and only if) $r \in\left\{1, \frac{n}{n-1}, \frac{n}{n-2}, \ldots, n\right\}$, it follows that if $\frac{n}{n-j}$ is a solution to $P^{j}$, for some $j>0$, then $U_{n}^{j-1}\left(r_{j-1}^{*}\right) \geq U_{n}^{j-1}\left(\frac{n}{n-j}\right)=U_{n}\left(\frac{n}{n-j}\right)>U_{n}^{j}\left(\frac{n}{n-j}\right)$. Thus, in order to find a maximum in $[1, R]$ we can concentrate on those $j$ s for which $\frac{n}{n-j}$ is not a solution to $P^{j}$; let $J$ be such a set. Let $r^{*}$ be a solution to $\max _{r \in\left\{r_{j}^{*}: j \in J\right\}} U_{n}(r)$.

We claim that $r^{*}$ solves the problem (49). Letting $\tilde{I}_{j}=\left(\frac{n}{n-j+1}, \frac{n}{n-j}\right]$ for $j=1, \ldots, n-1$, $\tilde{I}_{n}=(n, \infty)$ and $\tilde{I}_{0}=\{1\}$, we can separate it into the following problems, denoted $\tilde{P}^{j}$ :

$$
\max _{r} U_{n}^{j}(r)=E_{t} E_{i}\left[\frac{n-j}{(n-1) i+1} u(r)+(1-t) u\left(\max \left\{\frac{R(n-r(n-1) i}{n-(n-1) i}\right\}\right)\right]
$$

subject to $r \in \tilde{I}_{j} \cap W_{n}^{j} \cap D_{n}$.

Since for $j \in J$ the solution does not involve $\frac{n}{n-j}, \tilde{P}^{j}$ has the same solutions as $P^{j}$. Hence, $r^{*}$ is a solution to program (49).

Lemma 12 For all $n$, there is a symmetric equilibrium in which all consumers deposit.

Proof. The strategies are: the bank offers $r^{*}$, and the consumers choose $d^{*}(r)=1$ if $r \in D_{n} \cap W_{n}$ and 0 otherwise, $w^{*}(r, d, 2)=0$ if $r \in D_{n} \cap W_{n}$ and $d=1$ and 1 otherwise, and $w^{*}(r, d, 1)=1$ for all $(r, d)$.

\section{A.4 A Positive Probability of an Equilibrium Bank Run}

In this section we prove Proposition 1. By Lemma 12, there is an equilibrium in which all consumers deposit for all $n \in \mathbb{N}$ and all functions $f$.

Let $\tau$ be as in Lemma 7 and let $\bar{t}>\tau$ and $f \in \mathcal{F}_{\bar{t}}$. Then, there is $\tilde{r}>1 / \bar{t}$ such that $U(\tilde{r})>U(r)$ for all $r \in[1,1 / \tilde{t}]$ and $W L(\tilde{r})>W R(\tilde{r})$. Then, by Lemmas 4 and 5 , it follows 
that $U_{n}(\tilde{r})>U_{n}(r)$ for all $r \in[1,1 / \bar{t}]$ and $W L_{n}(\tilde{r})>W R_{n}(\tilde{r})$ if $n$ is large. Thus, $r_{n}^{*}>1 / \bar{t}$. Hence, the bank fails with a probability given by

$$
\int_{0}^{\bar{t}} f(t)\left(\sum_{i \in S_{n}: i>1 / r_{n}^{*}} p_{n, t}(i)\right) \mathrm{d} t>0
$$

since $\sum_{i \in S_{n}: i>1 / r^{*}} p_{n, t}(i)>0$ if $t>0$.

\section{A.5 A Limit Result on the Probability of an Equilibrium Bank Run}

Here we prove proposition 2. First, we claim that the sequence of interest rates is bounded away from $\bar{r}$.

Claim 3 There exists $\varepsilon>0$ such that $r_{n}^{*} \geq \bar{r}+\varepsilon$ for all $n$.

Proof. Suppose not. Then there is a subsequence $\left\{r_{n_{k}}^{*}\right\}$ such that $r_{n_{k}}^{*} \rightarrow \bar{r}$. Let $\tilde{r}$ be as in Lemma 7 . Then, $U_{n_{k}}\left(r_{n_{k}}^{*}\right) \geq U_{n_{k}}(\tilde{r})$ for all $k$ and Lemma 4 imply that $U(\bar{r}) \geq U(\tilde{r})$, a contradiction.

Hence, $\left\{i>\frac{1}{\bar{r}+\varepsilon}\right\} \subseteq\left\{i>\frac{1}{r_{n}^{*}}\right\}$, and so

$$
\gamma_{n}=\int_{0}^{\bar{t}} f(t)\left(\sum_{i \in S_{n}: i>1 / r_{n}^{*}} p_{n, t}(i)\right) \mathrm{d} t \geq \int_{0}^{\bar{t}} f(t)\left(\int_{0}^{1} \chi_{(1 /(\bar{r}+\varepsilon), 1]} \mathrm{d} p_{n, t}\right) \mathrm{d} t .
$$

Let $\delta>0$ and $g:[0,1] \rightarrow[0,1]$ be a continuous function satisfying $g=\chi_{(1 /(\bar{r}+\varepsilon), 1]}$ in $[0,1 /(\bar{r}+\varepsilon)] \cup[1 /(\bar{r}+\varepsilon)+\delta, 1]$. Then,

$$
\int_{0}^{\bar{t}} f\left(\int_{0}^{1} g \mathrm{~d} p_{n, t}\right) \mathrm{d} t \rightarrow \int_{0}^{\bar{t}} f g \geq 1-F(1 /(\bar{r}+\varepsilon)+\delta) .
$$

Since $g \leq \chi_{(1 /(\bar{r}+\varepsilon), 1]}$, we obtain

$$
\underset{n}{\liminf } \int_{0}^{\bar{t}} f(t)\left(\int_{0}^{1} \chi_{(1 /(\bar{r}+\varepsilon), 1]} \mathrm{d} p_{n, t}\right) \mathrm{d} t \geq 1-F(1 /(\bar{r}+\varepsilon)+\delta)
$$

since this holds for all $\delta>0$, it follows that

$$
\liminf _{n} \int_{0}^{\bar{t}} f(t)\left(\int_{0}^{1} \chi_{(1 /(\bar{r}+\varepsilon), 1]} \mathrm{d} p_{n, t}\right) \mathrm{d} t \geq 1-F(1 /(\bar{r}+\varepsilon)),
$$

by letting $\delta \rightarrow 0$. Hence, $\liminf _{n} \gamma_{n} \geq 1-F(1 /(\bar{r}+\varepsilon))>0$, as desired. 


\section{References}

[1] Adão, B., and T. Temzelides (1998) "Sequential Equilibrium and Competition in a Diamond-Dybvig Banking Model," Review of Economic Dynamics, 1, 859-877.

[2] Allen, F., and D. Gale (1998) "Optimal Financial Crises," The Journal of Finance, 53, $1245-1284$

[3] Amaral, P., and G. Carmona (2004) "Bank Runs caused by Great Depressions," Southern Methodist University and Universidade Nova de Lisboa.

[4] Andolfatto, D., and E. Nosal (2006) "Moral Hazard, Sequential Service, and the Design of Bank Liabilities," Simon Frasier University and Federal Reserve Bank of Cleveland.

[5] Barlo, M., and G. Carmona (2003) "Strategic Behavior in Non-Atomic Games: A Refinement of Nash Equilibrium," Sabancı University and Universidade Nova de Lisboa.

[6] Calomiris, C., and J. Mason (2003) "Fundamentals, Panics, and Bank Distress During the Depression," The American Economic Review, 93, 1615-1647.

[7] Carmona, G. (2003) "Nash and Limit Equilibria of Games with a Continuum of Players," Universidade Nova de Lisboa.

[8] Diamond, D., and P. Dybvig (1983) "Bank Runs, Deposit Insurance, and Liquidity," Journal of Political Economy, 91, 401-419.

[9] Ennis, H., and T. Kiester (2003) "Economic Growth, Liquidity, and Bank Runs," Journal of Economic Theory, 109, 220-245.

[10] Freund, J., (1992) Mathematical Statistics, Third Edition, London: Prentice Hall.

[11] Goldstein, I., and A. Pauzner (2005) "Demand-Deposit Contracts and the Probability of Bank Runs," The Journal of Finance, 60, 1293-1327.

[12] Gorton, G. (1988) "Banking Panics and Business Cycles," Oxford Economic Papers, 40, 751-781. 
[13] Green, E., and P. Lin (2000) "Diamond and Dybvig's Classic Theory of Financial Intermediation: What's Missing?," Federal Reserve Bank of Minneapolis Quarterly Review, $24,3-13$.

[14] Green, E., and P. Lin (2002) "Implementing Efficient Allocations in a Model of Financial Intermediation," Journal of Economic Theory, 109, 1-23.

[15] Kaminsky, G. and C. Reinhart (1999) "The Twin Crises: The Causes of Banking and Balance-of-Payments Problems," American Economic Review, 89, 473-500.

[16] Hildenbrand, W. (1971) "Random Preferences and Equilibrium Analysis," Journal of Economic Theory, 3, 414-429.

[17] Jacklin, C. (1987) "Demand Deposits, Trading Restrictions, and Risk Sharing," in E. Prescott, and N. Wallace, eds., Contractual Arrangements for Intertemporal Trade, Minneapolis: University of Minnesota Press.

[18] Meirowitz, A. (2005) "Informational Party Primaries and Strategic Ambiguity," Journal of Theoretical Politics, 17, 107-136.

[19] Peck, J., and K. Shell (2002) "Equilibrium Bank Runs," Journal of Political Economy, $111,103-123$.

[20] Peck, J., and K. Shell (2003) "Bank Portfolio Restrictions and Equilibrium Bank Runs," The Ohio State University and Cornell University.

[21] Rudin, W. (1976) Principles of Mathematical Analysis, Singapore: McGraw-Hill.

[22] Wallace, N. (1988) "Another Attempt to Explain an Illiquid Banking System: The Diamond-Dybvig Model with Sequential Service Taken Seriously," Federal Reserve Bank of Minneapolis Quarterly Review, 12, 3-16.

[23] Wallace, N. (1990) "A Banking Model in Which Partial Suspension is Best," Federal Reserve Bank of Minneapolis Quarterly Review, 14, 11-23. 
[24] Wheeden, R. and A. Zygmund, (1977) Measure and Integral: An Introduction to Real Analysis, New York: Dekker.

[25] Williamson, S. (1988) "Liquidity, Banking, and Bank Failures," International Economic Review, 29, 25-43. 